difficulties, and 5 had persisting absence seizures, some with asymmetric interictal EEGs. None had abnormal neurologic signs, and neuroimaging was unremarkable. (Chaix Y, Daquin G, Monteiro F et al. Absence epilepsy with onset before age three years: a heterogeneous and often severe condition. Epilepsia July 2003;44:944-949). (Respond: Dr Yves Chaix, Centre Saint-Paul, Hopital Henri Gastaut, Marseille, France).

COMMENT. Early onset absence epilepsy is a rare heterogeneous condition with a generally poor outcome.

\title{
TEMPORAL LOBECTOMY FOR EPILEPSY WITH CONGENITAL PORENCEPHALY AND HIPPOCAMPAL SCLEROSIS
}

The clinical characteristics and surgical outcome of 6 patients with intractable epilepsy and coexisting extratemporal porencephaly and hippocampal sclerosis are presented from the University of Alabama, Birmingham, AL. Of 24 patients with congenital porencephaly and epilepsy, 6 had a temporal lobe epileptogenic focus, and temporal lobe resection was performed in 5. The mean age at surgery was 31 years (range, 15-42 years), and the time from onset of epilepsy was 27 years (range, 14-41 years). Mean age of seizure onset was 4.3 years (range, 6 months to 10 years). Porencephalic cyst volume was $11 \%$ of total intracranial volume (range, $1 \%$ to $32 \%$ ). Freedom from seizures occurred in all five patients, at mean follow-up of 47 months (range, 22-67 months). Antiepileptic drug therapy was continued but at lower doses. Hippocampal sclerosis was confirmed histopathologically. Children with congenital porencephaly and intractable epilepsy should be evaluated early, and temporal lobectomy should be considered if clinical, MRI, and EEG findings indicate a temporal lobe origin for seizures. (Burneo JG, Faught E, Knowlton RC et al. Temporal lobectomy in congenital porencephaly associated with hippocampal sclerosis. Arch Neurol June 2003;60:830-834). (Reprints: Ruben Kuzniecky MD, University of Alabama at Birmingham Epilepsy Center, Civitan International Research Center (CIRC) 312, 1719 Sixth Ave S, Birmingham, AL 35294).

COMMENT. A common ischemic cause for the congenital porencephalic cyst and hippocampal sclerosis is postulated, involving perinatal occlusion of the posterior cerebral artery. The most frequent origin of seizures associated with the dual pathology is the temporal lobe. The selective resection of the temporal epileptogenic focus might be considered as an alternative to hemispherectomy, which carries a higher morbidity in these patients.

\section{HEADACHE DISORDERS}

\section{QUALITY OF LIFE IN CHILDHOOD MIGRAINE}

The Pediatric Quality of Life Inventory (PQLI), Version 4.0 and a standardized headache assessment were completed by children and parents, in a survey of 572 consecutive patients (mean age, $11.4+/-3.6$ years) who presented with headaches at the Cincinnati Children's Hospital Medical Center, Ohio. Most (99\%) had a clinical diagnosis 
of migraine, $85 \%$ met migraine diagnostic criteria of the International Headache Society, and $40 \%$ had chronic daily headaches. The PQLI score for the group $(73.1+/-14.4)$ was lower than that for healthy norms $(83.0+/-14.8)$ and was lowest for children with chronic daily headaches $(70.5+/-15.5)$. The impairment of QOL in children with migraine was similar to that observed in children with arthritis and cancer, affecting school and emotional functioning. (Powers SW, Patton SR, Hommel KA, Hershey AD. Quality of life in childhood migraines: clinical impact and comparison to other chronic illnesses. Pediatrics July 2003;112:e1-e5). (Reprints: Scott W Powers PhD, Headache Center, Division of Psychology, Cincinnati Children's Hospital, MLC, D-3015, 3333 Burnet Ave, Cincinnati, OH 45229). E-mail:Scott.Powers@cchmc.org).

COMMENT. The prevalence of childhood migraine is estimated at about $11 \%$, more than twice that reported in earlier studies. Environmental factors, including school, social, and family tensions, are cited as factors in the increased prevalence of migraine noted in the last decade. (see Progress in Pediatric Neurology III, PNB Publishers, 1997;pp187-190). A similar increase in migraine prevalence is noted in adult populations. With the increased consumption of snack foods, caffeine-containing beverages, and alcohol among children and adolescents, diet as a cause or precipitant of migraine is receiving increased attention (Millichap JG, Yee MM. Pediatr Neurol Jan 2003;28:9-15). Headaches, especially chronic daily headaches, cause a pattern of disability and impairment in quality of life similar to that in children with rheumatoid arthritis or cancer. Children with migraine report more impairment in school and emotional functioning than those with other chronic diseases.

\section{DEGENERATIVE DISORDERS}

\section{NITROUS OXIDE TOXICITY WITH METHYLENETETRAHYDRO- FOLATE REDUCTASE DEFICIENCY}

The neurological deterioration and death of a child with methylenetetrahydrofolate reductase (MTHFR) deficiency following anesthetization with nitrous oxide are reported from the University of Wisconsin Medical School, Madison, WI, and McGill University, Montreal. The child appeared normal until 3 months of age, when a mass in the left leg developed. The patient's father, an uncle, and a sibling had elevated levels of homocysteine, and received high-dose vitamin B supplements. Twenty five days after resection of an infantile fibrosarcoma under halothane and $60 \%$ nitrous oxide anesthesia, the patient was readmitted because of seizures and episodic apnea. He was severely hypotonic, and areflexic. CT of brain showed generalized cerebral atrophy. Urine was positive for homocystine, but negative for organic acids and methylmalonic acid. Plasma homocystine was elevated, methionine level was low, vitamin $\mathrm{B}_{12}$ level normal, serum folate low normal, and csf folate normal. The patient died at 130 days of age (46 days after operation) with respiratory arrest. Autopsy revealed cerebral atrophy and severe demyelination, with astrogliosis in the midbrain, medulla, and cerebellum. Values for MTHFR activity in cultured fibroblasts were low. The child and his affected relatives were heterogeneous for a novel mutation which causes substitution of isoleucine for methionine. The mutation was transmitted from a paternal chromosome. Nitrous oxide inactivates 